\title{
«Tout en couleur» ou "seuls les médecins voient tout en noir»
}

\section{Yvonne Gilli}

Dr méd., membre du Comité central de la FMH, département Numérisation / eHealth

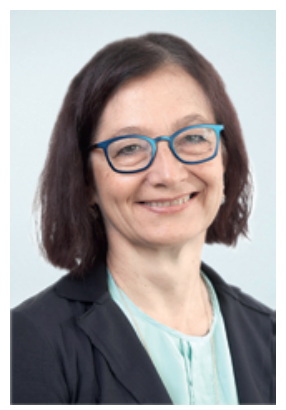

Suite à l'échec d'une solution uniforme lors de la Conférence de radiodiffusion à Stockholm et de la tentative de médiation de l'assemblée plénière du Comité Consultatif International des Radiocommunications à Oslo en vue d'une norme unique, le Conseil fédéral a décidé il y a 50 ans, le 15 août 1967, d'introduire le système PAL. Vous l'avez compris, il est question ici de l'introduction de la télévision couleur en Suisse. Cette décision a eu d'importantes répercussions, du moins pour les téléspectateurs de Suisse romande. Comme la France avait, pour des raisons politiques, opté pour le système SECAM («Séquentiel couleur à mémoire» ou "Système élégant contre l'Amérique»), les téléspectateurs romands ont été contraints d'acquérir un récepteur multinorme pour recevoir les programmes français en plus des programmes suisses en couleur. Dans son co-rapport, le conseiller fédéral de l'époque Roger Bonvin a déclaré regretter vivement cette situation, mais que celle-ci était inévitable en raison des conditions techniques à l'étranger.

50 ans plus tard, le 5 juillet 2017, le Département fédéral de l'intérieur a ouvert la procédure de consultation concernant la révision de l'ordonnance sur le dossier électronique du patient pour l'introduction des formats d'échange électroniques. Avec la reprise des prescrip-

Les médecins qui désirent "recevoir» les données structurées devront acquérir un récepteur adapté.

tions, aussi bien sur le plan technique que du contenu, pour l'échange d'informations structurées dans le dossier électronique du patient (DEP), le DEP tente de passer pour la première fois à la couleur. Ici aussi, la décision de la Confédération n'est pas sans conséquence: les médecins qui souhaitent «recevoir» ces données structurées devront acquérir un récepteur adapté.

S'il n'est pas inhabituel qu'une ordonnance soit révisée peu après l'entrée en vigueur d'une loi-cadre, les nombreuses interfaces du DEP avec la cybersanté et différentes réglementations (nouvelles ou déjà existantes) concernant notamment l'intervention tarifaire (LAMal) et les lois sur les professions médicales, les produits thérapeutiques et la protection des données rendent la situation particulièrement compliquée.

Dans le projet de révision totale de la loi sur la protection des données (LPD) avec la reprise de l'ordonnance (EU) 2016/679, le corps médical est soumis à des obligations plus strictes que nécessaire: les médecins sont en

Un manque de coordination du droit fédéral aura pour conséquence l'établissement de solutions différentes sur le marché.

effet tenus d'informer les destinataires des données, c'est-à-dire les patients et les spécialistes chargés du suivi, de toute rectification, suppression, ou destruction des données, de toute violation de la protection des données, etc., sous peine de sanctions.

La façon dont le législateur entend mettre en œuvre cette réglementation dans le contexte du DEP n'est pas claire: comme la communication du dossier n'est pas adressée à une personne en particulier, le médecin ne peut pas savoir qui, parmi les professionnels de santé, consultera ces données.

Désormais, les exigences relatives à la prescription (électronique) doivent être réglées à l'art 50, révision du train d'ordonnances sur les produits thérapeutiques, qui suivent les principes de l'annexe à la Directive d'exécution 2012/52/EU. Du point de vue de la numérisation du système de santé, et plus précisément de la signature électronique, ces dispositions sont en contradiction avec le DEP. De plus, elles passent sous silence les problèmes centraux du soutien électronique au processus de médication.

Le rapport de la Direction générale des PTT du 31 mai 1967 précise que l'absence d'une norme européenne contribue à augmenter les coûts pour les téléspectateurs. Un manque de coordination du droit fédéral aura pour conséquence l'établissement de solutions différentes sur le marché, avec des répercussions sur la cybersanté et, au final, sur le DEP puisqu'il faudra un récepteur adapté pour tout. Certains voient déjà noir. 